\title{
A Theoretical Characterization of Covalency in Rare Earth Complexes through Their Absorption Electronic Properties: $f-f$ Transitions
}

\author{
Laurence Petit,,$+\neq$ Alain Borel,, Claude Daul,, ,ll Pascale Maldivi, ${ }^{,, \dagger}$ and Carlo Adamo ${ }^{\ddagger}$ \\ Laboratoire de Reconnaissance Ionique, DRFMC/LCIB (UMR_E 3 CEA-UJF), CEA-Grenoble, 17 \\ rue des Martyrs, F-38054 Grenoble Cedex 9, France, Laboratoire d'Electrochimie et de Chimie \\ Analytique, CNRS UMR-7575, École Nationale Supérieure de Chimie de Paris, 11 rue P. et M. \\ Curie, F-75231 Paris Cedex 05, France, Institut des Sciences et Ingénierie Chimiques, Ecole \\ Polytechnique Fédérale de Lausanne, EPFL-BCH, CH-1015 Lausanne, Switzerland. and \\ Département de Chimie, Université de Fribourg, Pérolles, CH-1700 Fribourg, Switzerland
}

\begin{abstract}
Experimental uncertainties concerning the coordination mode of trivalent plutonium in concentrated $\mathrm{LiCl}$ have led us to theoretically evaluate the $f-f$ transitions of a series of rare earth aquo and chloro complexes. The calculation of $\operatorname{Pr}(I I I), U(I I I), N p(I I I)$, and $\mathrm{Pu}(\mathrm{III})$ systems' spectra was undertaken using the LFDFT (ligand field density functional theory) route that combines the backgrounds of ligand field (LF) theory with Kohn-Sham orbitals. LF parameters are fitted to previous DFT calculations, thus preventing the use of empirical data. The $f-f$ transitions values are globally well predicted, but the lack of accurate experimental references can sometimes hinder reliable comparisons. Despite this, the nephelauxetic effect from aquo to chloro complexes is clearly observed through both spectral red shifts and the decrease in $F_{2}$, the Slater-Condon parameter. Accordingly, this work provides the first theoretical characterization of covalency in trivalent $f$ elements through their electronic spectra.
\end{abstract}

\section{Introduction}

Research into chloride complexes of trivalent lanthanides and actinides has received increasing attention in the past decade because of their implication in the long-term management of spent nuclear fuel. In addition to their involvement in hydrometallurgical and pyrochemical processes, they are also assumed to be present in geological salt formations, thereby raising questions concerning their transport properties and their interaction with the environment. For this reason, numerous studies have focused on the determination of their stability constants, ${ }^{1-3}$ but inconsistencies between conclusions drawn from different experimental techniques suggest

* To whom correspondence should be addressed. Phone: +33 4.38.78.53.03. Fax: +33 4.38.78.50.90. E-mail: pascale.maldivi@cea.fr (P.M.). Phone: +41 26.300.8741. Fax: +41 26.300.9738. E-mail: claude.daul@unifr.ch (C.D.).

${ }^{\dagger}$ Laboratoire de Reconnaissance Ionique.

École Nationale Supérieure de Chimie de Paris.

$\S$ Ecole Polytechnique Fédérale de Lausanne.

"Université de Fribourg.

(1) Sillen, L. G.; Martell, A. E. Stability Constants of Metal-Ion Complexes; Special Publication 17; The Chemical Society: London, 1964; Special Publication 25; The Chemical Society: London, 1971.

(2) Fanghänel, T.; Kim, J. I.; Klenze, R.; Kato, Y. J. Alloys Compd. 1995, 225,308 .

(3) Chopin, G. R. J. Alloys Compd. 1997, 249, 9. that the coordination chemistry of these compounds is still an open question.

In the 1960s, Shiloh et al. studied the evolution of the UV-vis spectra of several actinides as a function of chloride concentration. ${ }^{4,5}$ They showed that neptunium and plutonium exhibit a strong characteristic absorption in concentrated lithium chloride attributed to a $5 \mathrm{f}^{n} \rightarrow 5 \mathrm{f}^{n-1} 6 \mathrm{~d}^{1}$ transition. Interestingly, such behavior was not observed in dilute $\mathrm{HCl}$, for the aquo complex. The authors therefore attributed this difference to the coordination of two chlorides.

More recently, Allen et al., ${ }^{6,7}$ used EXAFS spectroscopy (extended X-ray absorption fine structure) under similar experimental conditions. They globally agreed with the conclusions of Shiloh et al., observing that only water molecules were coordinated in dilute $\mathrm{HCl}$, while both chlorides and water were found in concentrated $\mathrm{LiCl}$. In contrast, even at a very high concentration of $\mathrm{LiCl}([\mathrm{LiCl}]$ $=12.3 \mathrm{M}), \mathrm{Pu}^{3+}$ showed no inner-sphere chloride complex-

(4) Shiloh, M.; Marcus, Y. J. Inorg. Nucl. Chem. 1966, 28, 2725

(5) Shiloh, M.; Marcus, Y. Isr. J. Chem. 1965, 3, 123.

(6) Allen, P. G.; Bucher, J. J.; Shuh, D. K.; Edelstein, N. M.; Reich, T. Inorg. Chem. 1997, 36, 4676.

(7) Allen, P. G.; Bucher, J. J.; Shuh, D. K.; Edelstein, N. M.; Craig, I. Inorg. Chem. 2000, 39, 595. 
ation but did show significant dehydration because of the decrease in water activity. Unexpectedly, only 5 water molecules were observed in the first coordination sphere. This result is all the more surprising given that, for nearby elements (americium and curium), chloride complexation has been characterized by several methods., ${ }^{2,7}$ To our knowledge however, no EXAFS data on their neptunium(III) counterparts is available. Even so, it is well-known that experiments on $\mathrm{Pu}(\mathrm{III})$ are extremely difficult to carry out because of its fast and easy oxidation. In addition, EXAFS can be seriously affected by various limitations such as its model dependency.

The aim of our work is therefore to theoretically reproduce the electronic absorption spectra of the aquo and chloro complexes of some f-elements and to analyze the influence of inner-shell ligands on metal-centered bands. This article is the first step toward this goal and examines the $\mathrm{f}-\mathrm{f}$ transitions of complexes of $\mathrm{U}(\mathrm{III}), \mathrm{Np}$ (III), and $\mathrm{Pu}(\mathrm{III})$ because their UV-vis spectra, obtained by Shiloh and coworkers, ${ }^{4,5}$ are available. In practice, the oxidation state + III is the most stable for heavy actinides, such as americium and curium, in solution and is thus involved in spent nuclear fuel. For such compounds, the diffuse character of their $5 \mathrm{f}$ orbitals is expected to make them environment sensitive. In contrast, lanthanide $\mathrm{f}-\mathrm{f}$ transitions should be far less affected. The Pr(III) complex was thus referred as a benchmark. Such a study prepares the ground for a second step focusing on the $\mathrm{f}-\mathrm{d}$ transitions.

Within this framework, the relevance of the LFDFT (ligand field density functional theory) route has been shown several times. ${ }^{8,9}$ It is based on the well-known and commonly used ligand field theory, but the inherent parameters of this approach are fitted to DFT calculations instead of experimental data, making LFDFT a nonempirical method. The total Hamiltonian of the system is thus determined on the basis of Kohn-Sham orbitals. As in a configuration interaction (CI) procedure, this Hamiltonian is diagonalized, and all the possible multiplets are generated as linear combinations of Slater determinants.

The interest of our study is thus 2-fold. First, our approach provides a fast and reliable tool for assessing $\mathrm{f}-\mathrm{f}$ transitions and consistently describing covalency effects, whereas the monodeterminantal TDDFT route still fails. Second, theoretical calculations help to shed light on a major experimental issue. Beyond clarification of the contradiction concerning $\mathrm{Pu}$ (III) results, our study is one of the first theoretical studies ${ }^{10}$ to provide insights into the covalency of trivalent f elements through their spectral properties.

\section{LFDFT Model}

We focus herein on the general procedure of the LFDFT model and refer the reader to refs 11 and 12 for more details on this theory. Three main steps can actually be considered.

(8) Atanasov, M.; Daul, C.; Gudel, H. U.; Wesolowski, T. A.; Zbiri, M. Inorg. Chem. 2005, 44, 2954.

(9) Atanasov, M.; Baerends, E. J.; Baettig, P.; Bruyndonckx, R.; Daul, C.; Rauzy,C.; Zbiri, M. Chem. Phys. Lett. 2004, 399, 433.

(10) Gutierrez, F.; Rabbe, C.; Poteau, R.; Daudey, J. P. J. Phys. Chem. A $\mathbf{2 0 0 5}, 109,4325$ (i) The first part consists of DFT calculations. After determination of the ground state geometry from experimental data or from an optimization, an average of configuration (AOC) is performed. Each of the 7 orbitals with predominant $\mathrm{f}$ character is equally filled with $n / 7$ electrons, the metal being $\mathrm{f}^{n}$. For instance, the $5 \mathrm{f}\left(\mathrm{Pu}^{\mathrm{III}}\right)$ levels are then occupied with 5/7 electrons. In this manner, we ensure a good starting point by imposing a spherical symmetry, as in an atomic calculation. This is indeed used further when approximating the electronic repulsion with atomic Slater-Condon parameters (see step 2). The resulting Kohn-Sham orbitals are kept frozen and are used to generate all the possible determinants (microstates) stemming from the $\mathrm{f}^{n}$ configuration $\left(C_{14}^{n}\right.$ on the whole). Their corresponding energies will provide the 1 st order (diagonal) contribution to the CI matrix. The off-diagonal matrix elements are obtained through ligand-field projection onto the singledeterminant energies as mentioned below.

(ii) The global Hamiltonian of the system is then classically divided into 3 terms: the electronic repulsion Hamiltonian, $H_{\mathrm{ER}}$, the spin-orbit coupling Hamiltonian, $H_{\mathrm{SO}}$, and the ligand field Hamiltonian, $H_{\mathrm{LF}}$ (the kinetic energy and the electron/nucleus interaction are not written here for clarity). In ligand field theory, each of these terms is parametrized

$$
\begin{array}{r}
H=H_{\mathrm{ER}}+H_{\mathrm{SO}}+H_{\mathrm{LF}}=a_{1} F_{0}+a_{2} F_{2}+a_{3} F_{4}+a_{4} F_{6}+a_{5} \zeta+ \\
a_{6} h_{11}+\ldots+a_{12} h_{77}
\end{array}
$$

where the Slater-Condon parameters from the electronic repulsion are termed $F_{k, k=0,2,4,6}, \zeta$ is the spin-orbit coupling constant, and $h_{i, i=1,2, \ldots, 7}$ are the 7 diagonal ligand field parameters. Thus, one only needs to calculate the above-listed parameters to get their corresponding Hamiltonians, and therefore the global Hamiltonian, $H$. Their values are collected within a so-called $\vec{P}$ vector which is then fitted to the previous DFT calculations performed at step 1 (Note that the nondiagonal ligand field parameters can be obtained from the composition of the corresponding KS orbitals if needed). ${ }^{11,12}$ Equation 1 can indeed be rewritten in matrix form

$$
\vec{E}(\mathrm{SD})=A \vec{P}
$$

where $\boldsymbol{A}=\left[\left\langle\mathrm{SD}_{i}|H| \mathrm{SD}_{j}\right\rangle\right]$ contains all the $a_{i i}$ coefficients and is deduced from Slater rules. The previous system is overdetermined and is solved with a least-squares fit, giving all the needed parameters

$$
\vec{P}=\left(A^{t} A\right)^{-1} A^{t} \vec{E}
$$

The consistency between the DFT energies values from step 1 and those calculated from the fitted parameters (step 2) proves the quality of our results, with a mean square deviation from 30 to 90 $\mathrm{cm}^{-1}$ versus the $80-120 \mathrm{~cm}^{-1}$ value usually expected (Table 1).

In practice, the spin-orbit coupling constant, $\zeta$, is obtained through a wave function optimization of the free ion including spin-orbit coupling. Two levels $\left(f_{5 / 2}\right.$ and $\left.f_{7 / 2}\right)$ logically come from this calculation, and their energies provide the $\xi$ value through the Landé formula

$$
\Delta E=E\left(\mathrm{f}_{7 / 2}\right)-E\left(\mathrm{f}_{5 / 2}\right)=\frac{7 \xi}{2}
$$

(iii) The global Hamiltonian is finally inferred from the sum of each contribution. The off-diagonal elements of the CI matrix are obtained from the best-fit $\vec{P}$ values using standard ligand field

(11) Atanasov, M.; Daul, C.; Rauzy, C. Chem. Phys. Lett. 2003, 367, 737.

(12) Atanasov, M.; Daul, C.; Rauzy, C. Structure and Bonding; SpringerVerlag: New York, 2003; Vol. 106, p 97. 
Table 1. Mean Square Deviation (MSD) on Fitted Slater-Condon, Spin-Orbit Coupling, and Ligand Field Paramaters $\left(\mathrm{cm}^{-1}\right)$

\begin{tabular}{|c|c|c|c|c|}
\hline & $\operatorname{Pr}^{3+}$ & $\mathrm{U}^{3+}$ & $\mathrm{Np}^{3+}$ & $\mathrm{Pu}^{3+}$ \\
\hline \multirow[t]{2}{*}{ MSD } & 80.3 & 41.4 & 34.4 & 29.8 \\
\hline & {$\left[\mathrm{Pr}\left(\mathrm{H}_{2} \mathrm{O}\right)_{9}\right]^{3+}$} & {$\left[\mathrm{U}\left(\mathrm{H}_{2} \mathrm{O}\right)_{9}\right]^{3+}$} & {$\left[\mathrm{Np}\left(\mathrm{H}_{2} \mathrm{O}\right)_{9}\right]^{3+}$} & {$\left[\mathrm{Pu}\left(\mathrm{H}_{2} \mathrm{O}\right)_{9}\right]^{3+}$} \\
\hline \multirow[t]{2}{*}{ MSD } & 80.6 & 45.4 & 36.4 & 30.9 \\
\hline & $\begin{array}{c}{\left[\mathrm{Pr}(\mathrm{Cl})_{2}\right.} \\
\left.\left(\mathrm{H}_{2} \mathrm{O}\right)_{7}\right]^{+}\end{array}$ & $\begin{array}{c}{\left[\mathrm{U}(\mathrm{Cl})_{2}\right.} \\
\left.\left(\mathrm{H}_{2} \mathrm{O}\right)_{7}\right]^{+}\end{array}$ & $\begin{array}{l}{\left[\mathrm{Np}(\mathrm{Cl})_{2}\right.} \\
\left.\left(\mathrm{H}_{2} \mathrm{O}\right)_{7}\right]^{+}\end{array}$ & $\begin{array}{c}{\left[\mathrm{Pu}(\mathrm{Cl})_{2}\right.} \\
\left.\left(\mathrm{H}_{2} \mathrm{O}\right)_{7}\right]^{+}\end{array}$ \\
\hline MSD & 87.7 & 48.7 & 37.8 & 32.2 \\
\hline
\end{tabular}

theory. The diagonalization of the CI matrix yields the multiplet energies. Assignments are then predicted from the expectation values of the $L, S$, and $J$ operators.

\section{Computational Details}

DFT calculations were performed with the ADF package (program release 2004.01), ${ }^{13-15}$ while we used the suite of programs implemented by A. Borel and C. Daul ${ }^{16}$ for the LFDFT part. Since experimental structures are not available for all complexes, geometries were optimized starting either from $\left[\mathrm{Pr}\left(\mathrm{H}_{2} \mathrm{O}\right)_{9}\right]^{3+}$ or $[\mathrm{Th}-$ $\left.(\mathrm{Cl})_{2}\left(\mathrm{H}_{2} \mathrm{O}\right)_{7}\right]^{2+}$ crystallographic structures. ${ }^{17-19}$ They were slightly modified to impose the closest symmetry, that is to say $C_{3 v}$ and $C_{2 v}$ for the aquo and chloro complexes, respectively. Coordination numbers were chosen in agreement with EXAFS, transport, X-ray diffraction, or even theoretical data., ${ }^{77,20-25}$ It is worth noting that the U(III) aquo complex can obviously seem surprising because of its easy oxidation to U(IV) with water molecules. Yet, several works have shown that it is possible to find hydrated U(III) complexes under particular experimental conditions. ${ }^{22,26-29}$

Trivalent rare earths generally experience a weak ligand field so the highest multiplicity was applied each time. As already reported, ${ }^{30,31}$ GGA (generalized gradient approximation) functionals produce metal-ligand distances that are too long with respect to that obtained within the local density approximation (LDA), whereas they are preferred for calculating electronic properties. Bad geometries may alter the absorption spectra so the following procedure was adopted. Geometries were first optimized with the LDA Vosko, Wilk, and Nusair functional ${ }^{32}$ to get structures as close as possible to experimental data. 7,17,20-25 The GGA Becke Perdew

(13) Baerends, E. J.; Ellis, D. E.; Ros, P. Chem. Phys. 1993, 2, 42.

(14) Boerrigter, P. M.; te Velde, G.; Baerends, E. J. Int. J. Quantum Chem. 1988, 33, 87.

(15) te Velde, G.; Baerends, E. J. Comput. Phys. 1992, 99, 84

(16) Programs are freely available on request to C. Daul.

(17) Chatterjee, A.; Masten, E. N.; Watson, K. J. Acta Crystallogr. B 1988 , $381,44$.

(18) Rogers, R. D. Lanthanide Actinide Res. 1989, 3, 71.

(19) Parker, D.; Dickins, R. S.; Puschmann, H.; Crossland, C.; Howard, J. A. K. Chem. Rev. 2002, 102, 1977.

(20) Matonic, J. H.; Scott, B. L.; Neu, M. P. Inorg. Chem. 2001, 40, 2638.

(21) David, F.; Vokhmin, V.; Ionova, G. J. Mol. Liq. 2001, 90, 45.

(22) David, F.; Fourest, B. New. J. Chem. 1997, 21, 167.

(23) Antonio, M. R.; Soderholm, L.; Williams, C. W.; Blandeau, J. P.; Bursten, B. E. Radiochim. Acta 2001, 89, 17.

(24) Conradson, S. D. Appl. Spectrosc. 1998, 52, 252A.

(25) Blandeau, J. P.; Zygmunt, S. A.; Curtiss, L. A.; Reed, D. T.; Bursten, B. E. Chem. Phys. Lett. 1985, 89, 3310.

(26) Drozdzynski, J. Inorg. Chim. Acta 1985, 109, 79.

(27) Zych, E.; Drozdzynski, J. Inorg. Chim. Acta 1986, 115, 219.

(28) Karbowiak, M.; Drozdzynski, J.; Janczak, J. Polyhedron 1996, 2, 241.

(29) Karbowiak, M.; Drozdzynski, J.; Gajek, Z. J. Alloys Compd. 2001, $323-324,678$

(30) Borel, A.; Helm, L.; Daul, C. A. E. Chem. Phys. Lett. 2004, 383, 584.

(31) Guillaumont, G. J. Phys. Chem. A 2004, 108, 6893.

(32) Vosko, S. H.; Wilk, L.; Nusair, M. Can. J. Chem. 1980, 58, 1200.
Table 2. Theoretical (ADF) and Experimental $\mathrm{M}-\mathrm{O}\left(\mathrm{H}_{2}\right)\left(d_{\mathrm{M}-\mathrm{O}}\right)$ and $\mathrm{M}-\mathrm{Cl}\left(d_{\mathrm{M}-\mathrm{Cl}}\right)$ Distances $^{a}$

\begin{tabular}{lcccccc}
\hline & & \multicolumn{2}{c}{ SVWN } & & \multicolumn{2}{c}{ experiment } \\
\cline { 3 - 4 } & symmetry & $d_{\mathrm{M}-\mathrm{O}}$ & $d_{\mathrm{M}-\mathrm{Cl}}$ & & $d_{\mathrm{M}-\mathrm{O}}$ & $d_{\mathrm{M}-\mathrm{Cl}}$ \\
\hline$\left[\mathrm{Pr}\left(\mathrm{H}_{2} \mathrm{O}\right)_{9}\right]^{3+}$ & $C_{3 v}$ & 2.53 & & & $2.51,{ }^{b} 2.51^{c}$ & \\
{$\left[\mathrm{Pr}(\mathrm{Cl})_{2}\left(\mathrm{H}_{2} \mathrm{O}\right)_{7}\right]^{+}$} & $C_{2 v}$ & 2.50 & 2.75 & & $(2.54)$ & $(2.87)$ \\
{$\left[\mathrm{U}\left(\mathrm{H}_{2} \mathrm{O}\right)_{9}\right]^{3+}$} & $C_{3 v}$ & 2.53 & & & $2.56^{d}$ & \\
{$\left[\mathrm{U}(\mathrm{Cl})_{2}\left(\mathrm{H}_{2} \mathrm{O}\right)_{7}\right]^{+}$} & $C_{2 v}$ & 2.52 & 2.76 & & & \\
{$\left[\mathrm{~Np}\left(\mathrm{H}_{2} \mathrm{O}\right)_{9}\right]^{3+}$} & $C_{3 v}$ & 2.51 & & & $2.52,{ }^{c} 2.48^{e}$ & \\
{$\left[\mathrm{~Np}(\mathrm{Cl})_{2}\left(\mathrm{H}_{2} \mathrm{O}\right)_{7}\right]^{+}$} & $C_{2 v}$ & 2.50 & 2.74 & & & \\
{$\left[\mathrm{Pu}\left(\mathrm{H}_{2} \mathrm{O}\right)_{9}\right]^{3+}$} & $C_{3 v}$ & 2.50 & & & $2.51,,^{c} 2.51, f$ & \\
& & & & & $2.50,{ }^{g} 2.49^{h}$ & \\
{$\left[\mathrm{Pu}(\mathrm{Cl})_{2}\left(\mathrm{H}_{2} \mathrm{O}\right)_{7}\right]^{+}$} & $C_{2 v}$ & 2.49 & 2.73 & & $(2.52)$ & $(2.82)$
\end{tabular}

${ }^{a}$ Values in parentheses correspond to expected values from comparison to nearby elements EXAFS data. ${ }^{7}{ }^{b}$ Transport, ref $21 .{ }^{c}$ X-ray diffraction, ref 17. ${ }^{d}$ EXAFS, ref $44 .{ }^{e}$ EXAFS, ref $23 .{ }^{f}$ EXAFS, ref $6 .{ }^{g}$ X-ray diffraction, ref 20. ${ }^{h}$ EXAFS, ref 24.

functional ${ }^{33,34}$ was considered for the rest of the calculations. An increase in the default values for the numerical integration grid (accint $=8.0$ ) was found to give an easier convergence, while still respecting the Aufbau principle. Scalar relativistic calculations have been carried out within the zero order relativistic approximation (ZORA). Adapted triple- $\zeta$ plus two polarization function STO basis sets were used for the description of the valence part of all atoms, while we kept their core frozen up to $4 \mathrm{~d} / 5 \mathrm{~d}$ for lanthanides/actinides and up to $2 \mathrm{p}$ for chlorines and $1 \mathrm{~s}$ for oxygen atoms.

\section{Results and Discussion}

Geometries. Theoretical versus experimental distances are collected in Table 2. All the metals studied belong to the early lanthanides or actinides, and hence, their coordination numbers hardly change. The first hydration number of 9 for trivalent $\mathrm{Pr}, \mathrm{U}, \mathrm{Np}$, and $\mathrm{Pu}$ has been shown by several experimental techniques. ${ }^{7,17,20-25}$ In contrast, chloro complexes have been less characterized, and some controversies still remain about the number of chlorides into the inner metal shell. Chosen coordinations were thus extrapolated from EXAFS results ${ }^{6,7}$ and so are the distances mentioned in parentheses in Table 2. On the whole, the metal-oxygen bond lengths are found in very good agreement with experimental values, with a mean error of $0.02 \AA$, while the experimental error does not exceed $0.003 \AA$. Distances to chlorides seem to be rather underestimated ( $\Delta d \approx 0.1 \AA$ ), but the lack of experimental values precludes any comparison. As already pointed out by Atanasov et al. for rare earth hexachloro complexes, ${ }^{8}$ DFT seems to overestimate metal/ ligand covalency in such systems, yielding metal-chloride distances that are too short. Optimized geometries indeed feature some mixing of $\mathrm{d}$ and $\mathrm{f}$ metal orbitals on $\mathrm{p}(\mathrm{Cl})$ that must account for the discrepancy found for the metalchloride distances. Yet, when the LFDFT procedure is performed, only levels with predominant $\mathrm{f}$ character are considered, where the presence of $\mathrm{p}(\mathrm{Cl})$ and $\mathrm{p}(\mathrm{O})$ is negligible (less than $2 \%$ per orbital and less than $5 \%$ on the whole). The influence on the $\mathrm{f}-\mathrm{f}$ transitions and parameters values should then be rather low. Note that moving from the SVWN to BP functional does not significantly improve this trend, while the distances to the water molecules increase

(33) Becke, A. D. Phys. Rev. A 1988, 38, 3098

(34) Perdew, J. P. Phys. Rev. B 1986, 33, 8822. 
Table 3. Calculated and Experimental Electronic States and Energy Levels of $\left[\operatorname{Pr}\left(\mathrm{H}_{2} \mathrm{O}\right)_{9}\right]^{3+}$ and $\left[\operatorname{Pr}\left(\mathrm{Cl}_{2}\left(\mathrm{H}_{2} \mathrm{O}\right)_{7}\right]^{+a}\right.$

\begin{tabular}{|c|c|c|c|}
\hline \multicolumn{2}{|r|}{ calculations } & \multicolumn{2}{|c|}{ experiments } \\
\hline $\begin{array}{c}\text { level } \\
\text { assignment }\end{array}$ & $\begin{array}{c}\text { spectral } \\
\text { region }\left(\mathrm{cm}^{-1}\right)\end{array}$ & $\begin{array}{c}\text { level } \\
\text { assignment }\end{array}$ & $\begin{array}{c}\text { spectral } \\
\text { region }\left(\mathrm{cm}^{-1}\right)^{9}\end{array}$ \\
\hline \multicolumn{4}{|c|}{$\left[\operatorname{Pr}\left(\mathrm{H}_{2} \mathrm{O}\right)_{9}\right]^{3+}$} \\
\hline${ }^{3} \mathrm{H}_{4}$ & $\begin{array}{l}0-1370 \\
(0-1222)\end{array}$ & ${ }^{3} \mathrm{H}_{4}$ & 200 \\
\hline${ }^{3} \mathrm{H}_{5}$ & $\begin{array}{l}2325-3620 \\
(2391-3525)\end{array}$ & ${ }^{3} \mathrm{H}_{5}$ & 2360 \\
\hline${ }^{3} \mathrm{H}_{6}$ & $\begin{array}{l}4600-5330 \\
(4742-5837 ; 6280)\end{array}$ & ${ }^{3} \mathrm{H}_{6}$ & 4500 \\
\hline${ }^{1} \mathrm{G}_{4}$ & $\begin{array}{l}5436,7115,7365, \\
7606(5970,6577-7633)\end{array}$ & ${ }^{1} \mathrm{G}_{4}$ & $\begin{array}{l}9100-11000, \\
9900\end{array}$ \\
\hline${ }^{3} \mathrm{~F}_{2}$ & $\begin{array}{l}7307,7430,7535 \\
(7651-7700,7813)\end{array}$ & ${ }^{3} \mathrm{~F}_{2}$ & 5200 \\
\hline${ }^{3} \mathrm{~F}_{3}$ & $\begin{array}{l}8670-9360 \\
(9073-9537)\end{array}$ & ${ }^{3} \mathrm{~F}_{3},{ }^{3} \mathrm{~F}_{4}$ & $\begin{array}{l}5800-7700, \\
6500\left({ }^{3} \mathrm{~F}_{3}\right)\end{array}$ \\
\hline${ }^{3} \mathrm{~F}_{4}$ & $\begin{array}{l}10875-11645 \\
(11279-11894)\end{array}$ & ${ }^{3} \mathrm{~F}_{4}$ & 6950 \\
\hline${ }^{1} \mathrm{D}_{2}$ & $\begin{array}{l}17635-19180 \\
(18508-20016)\end{array}$ & ${ }^{1} \mathrm{D}_{2}$ & $\begin{array}{l}16000-17500, \\
16840\end{array}$ \\
\hline${ }^{1} \mathrm{I}_{6}$ & $\begin{array}{l}22990-25045 \\
(24364-26322)\end{array}$ & ${ }^{1} \mathrm{I}_{6},{ }^{3} \mathrm{P}_{1}$ & $\begin{array}{l}21000-22100, \\
21500\end{array}$ \\
\hline${ }^{3} \mathrm{P}_{0}$ & $29554(31356)$ & ${ }^{3} \mathrm{P}_{0}$ & $\begin{array}{l}20200-21000, \\
20750\end{array}$ \\
\hline${ }^{3} \mathrm{P}_{1}$ & $\begin{array}{l}30385,30536 \\
(32139-32308)\end{array}$ & ${ }^{3} \mathrm{P}_{1}$ & 21300 \\
\hline${ }^{3} \mathrm{P}_{2}$ & $\begin{array}{l}31435-31710, \\
44884 \\
(33169-33485,47722)\end{array}$ & ${ }^{3} \mathrm{P}_{2}$ & $\begin{array}{l}22100-23500 \text {, } \\
22520\end{array}$ \\
\hline \multicolumn{4}{|c|}{$\left[\mathrm{Pr}(\mathrm{Cl})_{2}\left(\mathrm{H}_{2} \mathrm{O}\right)_{7}\right]^{+}$} \\
\hline${ }^{3} \mathrm{H}_{4}$ & $0-1340$ & & \\
\hline${ }^{3} \mathrm{H}_{5}$ & $2530-4650$ & & \\
\hline $\begin{array}{l}{ }^{3} \mathrm{H}_{6} \\
{ }_{1} \mathrm{G}_{4}\end{array}$ & $\begin{array}{l}4730-6030 \\
6385\end{array}$ & & \\
\hline $\begin{array}{l}{ }^{1} \mathrm{G}_{4} \\
{ }^{3} \mathrm{~F}_{2}\end{array}$ & $\begin{array}{l}6385 \\
6840-7020\end{array}$ & ${ }^{1} \mathrm{G}_{4}$ & 9930 \\
\hline${ }^{3} \mathrm{G}_{4}$ & $7130-8080$ & & \\
\hline${ }^{3} \mathrm{~F}_{3}$ & $8300-8820$ & & \\
\hline${ }^{3} \mathrm{~F}_{4}$ & $10690-11450$ & & \\
\hline${ }^{1} \mathrm{D}_{2}$ & $12640-17550$ & ${ }^{1} \mathrm{D}_{2}$ & 16807,17210 \\
\hline${ }^{1} I_{6}$ & $21320-23200$ & ${ }^{1} \mathrm{I}_{6},{ }^{3} \mathrm{P}_{1}$ & 21231,21213 \\
\hline${ }^{3} \mathrm{P}_{0}$ & 26670 & ${ }^{3} \mathrm{P}_{0}$ & 20704,20690 \\
\hline${ }^{3} \mathrm{P}_{1}$ & 27740 & & \\
\hline${ }^{3} \mathrm{P}_{2}$ & $\begin{array}{l}28545-28845, \\
43374\end{array}$ & ${ }^{3} \mathrm{P}_{2}$ & 22422,22578 \\
\hline
\end{tabular}

${ }^{a}$ Computed values for the free ion $\mathrm{Pr}^{3+}$ are indicated in parentheses for comparison with $\left[\operatorname{Pr}\left(\mathrm{H}_{2} \mathrm{O}\right)_{9}\right]^{3+}$. For $\left[\operatorname{Pr}\left(\mathrm{H}_{2} \mathrm{O}\right)_{9}\right]^{3+}, \mathrm{Pr}^{3+}$ aquo ion, ref 45 and 46; for $\left[\mathrm{Pr}(\mathrm{Cl})_{2}\left(\mathrm{H}_{2} \mathrm{O}\right)_{7}\right]^{+}$, assumed chloro complex in concentrated alcoholic solution, ref 47 and 48; and $\operatorname{Pr}(\mathrm{III})$ with chloride in water, ref 49.

by about $0.1 \AA$. This can be attributed to the absence of solvent molecules in the external solvation sphere; an effect that can be artificially compensated using LDA functionals.

Electronic Spectra. Computed spectra are listed in Tables 3-6. The $\mathrm{f}-\mathrm{f}$ transitions corresponding to each level assignment are split over a range of energies so the extrema of their spectral window are only given under the label "spectral region". Our values are globally in good agreement with experimental data. Wavenumbers and assignments are consistent until $10000-13000 \mathrm{~cm}^{-1}$ with errors below a few hundred per centimeter. Thereafter, as energies grow, the discrepancy steadily increases with a great overestimation of experimental values up to several thousand per centimeter, as for the ${ }^{3} \mathrm{P}$ bands of $\operatorname{Pr}(\mathrm{III})\left(|\Delta \sigma| \approx 6000 \mathrm{~cm}^{-1}\right)$. Indeed, whereas the first transitions are easily assigned, the assignments become increasingly difficult when moving to high energies because of a strong mixing between the various states.
Table 4. Calculated and Experimental Electronic States and Energy Levels of $\left[\mathrm{U}\left(\mathrm{H}_{2} \mathrm{O}\right)_{9}\right]^{3+}$ and $\left[\mathrm{U}(\mathrm{Cl})_{2}\left(\mathrm{H}_{2} \mathrm{O}\right)_{7}\right]^{+a}$

\begin{tabular}{|c|c|c|c|}
\hline \multicolumn{2}{|r|}{ calculations } & \multicolumn{2}{|c|}{ experiments } \\
\hline $\begin{array}{c}\text { level } \\
\text { assignment }\end{array}$ & $\begin{array}{c}\text { spectral } \\
\text { region }\left(\mathrm{cm}^{-1}\right)\end{array}$ & $\begin{array}{c}\text { level } \\
\text { assignment }\end{array}$ & $\begin{array}{c}\text { spectral } \\
\text { region }\left(\mathrm{cm}^{-1}\right)^{10}\end{array}$ \\
\hline \multicolumn{4}{|c|}{$\left[\mathrm{U}\left(\mathrm{H}_{2} \mathrm{O}\right)_{9}\right]^{3+}$} \\
\hline${ }^{4} \mathrm{I}_{9 / 2}$ & $\begin{array}{l}0-1100 \\
(0-465)\end{array}$ & & \\
\hline${ }^{4} \mathbf{I}_{11 / 2}$ & $\begin{array}{l}4442-5204 \\
(4222-4522)\end{array}$ & ${ }^{4} \mathbf{I}_{11 / 2}$ & $\begin{array}{l}4300-5100 \\
4560\end{array}$ \\
\hline${ }^{4} \mathbf{I}_{13 / 2}$ & $\begin{array}{l}7973-9034 \\
(7878-8263)\end{array}$ & ${ }^{4} \mathbf{I}_{13 / 2}$ & $\begin{array}{l}7550-8950 \\
8201\end{array}$ \\
\hline${ }^{2} \mathrm{H}_{9 / 2}$ & $\begin{array}{l}9906-10345 \\
(10505-10693)\end{array}$ & ${ }^{2} \mathrm{H}_{9 / 2},{ }^{4} \mathrm{~F}_{5 / 2}$ & $9000-10600$ \\
\hline${ }^{4} \mathbf{I}_{15 / 2}$ & $\begin{array}{l}11295-12587 \\
(11025-11709)\end{array}$ & $\begin{array}{l}{ }^{4} \mathrm{~F}_{7 / 2},{ }^{4} \mathrm{~S}_{3 / 2} \\
{ }^{4} \mathrm{G}_{5 / 2},{ }^{4} \mathrm{I}_{15 / 2}\end{array}$ & $10650-12900$ \\
\hline${ }^{4} \mathrm{G}_{7 / 2}$ & $\begin{array}{l}13755-14511 \\
(14888-15085)\end{array}$ & ${ }^{4} \mathrm{G}_{7 / 2}$ & $13000-14400$ \\
\hline${ }^{4} \mathrm{~F}_{5 / 2}$ & $\begin{array}{l}14663-14895 \\
(15105,15135)\end{array}$ & ${ }^{4} \mathrm{~F}_{5 / 2}$ & $6500-7500$ \\
\hline $\begin{array}{l}{ }^{2} \mathrm{H}_{11 / 2},{ }^{2} \mathrm{~K}_{13 / 2} \\
{ }^{2} \mathrm{P}_{1 / 2},{ }^{4} \mathrm{G}_{7 / 2} \\
{ }^{4} \mathrm{G}_{9 / 2},{ }^{2} \mathrm{I}_{11 / 2}\end{array}$ & $\begin{array}{l}15595-17520 \\
\left({ }^{2} \mathrm{H}_{11 / 2}=16123-16234,\right. \\
{ }^{2} \mathrm{~K}_{13 / 2}=17125-17683, \\
\left.{ }^{4} \mathrm{G}_{9 / 2}=17781-17976\right)\end{array}$ & $\begin{array}{l}{ }^{4} \mathrm{~F}_{9 / 2},{ }^{2} \mathrm{H}_{11 / 2}, \\
{ }^{2} \mathrm{~K}_{13 / 2},{ }^{4} \mathrm{D}_{3 / 2} \\
{ }^{2} \mathrm{P}_{1 / 2},{ }^{2} \mathrm{G}_{7 / 2} \\
{ }^{2} \mathrm{G}_{9 / 2}\end{array}$ & $14600-17800$ \\
\hline${ }^{4} \mathrm{G}_{7 / 2}$ & $\begin{array}{l}17699-18735 \\
(19106-19407)\end{array}$ & $\begin{array}{l}{ }^{4} \mathrm{D}_{5 / 2},{ }^{2} \mathrm{~K}_{15 / 2} \\
{ }^{4} \mathrm{D}_{3 / 2},{ }^{2} \mathrm{H}_{11 / 2} \\
{ }^{2} \mathrm{D}_{5 / 2}\end{array}$ & $17800-21000$ \\
\hline${ }^{2} \mathrm{I}_{13 / 2},{ }^{2} \mathrm{~K}_{15 / 2}$ & $\begin{array}{l}19266-20505 \\
\left({ }^{2} \mathrm{D}_{5 / 2}=15731-15771,\right. \\
\left.{ }^{2} \mathrm{~K}_{15 / 2}=20617-21024\right)\end{array}$ & & \\
\hline \multicolumn{4}{|c|}{$\left[\mathrm{U}(\mathrm{Cl})_{2}\left(\mathrm{H}_{2} \mathrm{O}\right)_{7}\right]^{+}$} \\
\hline${ }^{4} I_{9 / 2}$ & $0-726$ & & \\
\hline${ }^{4} I_{11 / 2}$ & $4368-4822$ & $J=11 / 2$ & $\sim 4600$ \\
\hline${ }^{4} I_{13 / 2}$ & $7871-8484$ & $J=13 / 2$ & $\sim 8200$ \\
\hline${ }^{4} \mathrm{~F}_{5 / 2}$ & $8864-9226$ & $J=5 / 2$ & $\sim 10200$ \\
\hline${ }^{2} \mathrm{H}_{9 / 2}$ & $9495-9954$ & $J=9 / 2$ & $\sim 9500$ \\
\hline${ }^{4} I_{15 / 2}$ & $10756-11880$ & $J=15 / 2$ & $\sim 11300$ \\
\hline${ }^{4} \mathrm{G}_{7 / 2}$ & $12099-12986$ & $\begin{array}{l}J=5 / 2,7 / 2 \\
J=7 / 2\end{array}$ & $\begin{array}{l}\sim 12200 \\
\sim 13500\end{array}$ \\
\hline $\begin{array}{l}{ }^{4} \mathrm{D}_{5 / 2},{ }^{4} \mathrm{G}_{7 / 2}, \\
{ }^{4} \mathrm{H}_{7 / 2}\end{array}$ & $13025-15200$ & & \\
\hline${ }^{2} \mathrm{H}_{11 / 2}$ & $15268-15959$ & & \\
\hline $\begin{array}{l}{ }^{4} \mathrm{G}_{9 / 2},{ }^{4} \mathrm{H}_{9 / 2}, \\
{ }^{2} \mathrm{~K}_{13 / 2},{ }^{4} \mathrm{G}_{7 / 2}, \\
{ }^{2} \mathrm{D}_{5 / 2},{ }^{2} \mathrm{~K}_{15 / 2}\end{array}$ & $15992-19055$ & $J=9 / 2$ & $\sim 14900$ \\
\hline & & $\begin{array}{l}J=3 / 2 \\
J=5 / 2,15 / 2\end{array}$ & $\begin{array}{l}\sim 16600 \\
\sim 18500\end{array}$ \\
\hline $\begin{array}{l}{ }^{2} \mathrm{~K}_{15 / 2} \\
{ }^{4} \mathrm{G}_{9 / 2}\end{array}$ & $\begin{array}{l}19111-19633 \\
20023-20272\end{array}$ & & \\
\hline
\end{tabular}

${ }^{a}$ Computed values for the free ion $\mathrm{U}^{3+}$ are indicated in parentheses for comparison with $\left[\mathrm{U}\left(\mathrm{H}_{2} \mathrm{O}\right)_{9}\right]^{3+}$. For $\left[\mathrm{U}\left(\mathrm{H}_{2} \mathrm{O}\right)_{9}\right]^{3+}$, ref 35 and 50; for $\left[\mathrm{U}(\mathrm{Cl})_{2}\left(\mathrm{H}_{2} \mathrm{O}\right)_{7}\right]^{+}$, chloro complex in $\mathrm{CH}_{3} \mathrm{OH}$ and DMF of $\mathrm{U}(\mathrm{III})$, ref 50 .

Actually, one should consider these discrepancies with care because of the possible inaccuracy of both the computed and experimental values. On one hand, discrepancies may come from several theoretical systematic errors already quoted in previous LFDFT studies. ${ }^{8}$ Calculations are all performed in a vacuum so solvent effects are not considered. The use of low symmetries, such as $C_{2 v}$ or $C_{3 v}$, may also alter the accuracy of the results since the number of integrals to be computed numerically is inversely proportional to the point group order. Although the CI procedure (see step 3 in Computational Details) allows us to explicitly treat the static correlation, this scheme introduces, at the same time, a part of dynamical correlation already taken into account in the DFT calculation that results in a double counting error. Finally, the approximate treatment of the exchange part is also responsible for the presence of spurious self-interaction 
Table 5. Calculated and Experimental Electronic States and Energy Levels of $\left[\mathrm{Np}\left(\mathrm{H}_{2} \mathrm{O}\right)_{9}\right]^{3+}$ and $\left[\mathrm{Np}(\mathrm{Cl})_{2}\left(\mathrm{H}_{2} \mathrm{O}\right)_{7}\right]^{+a}$

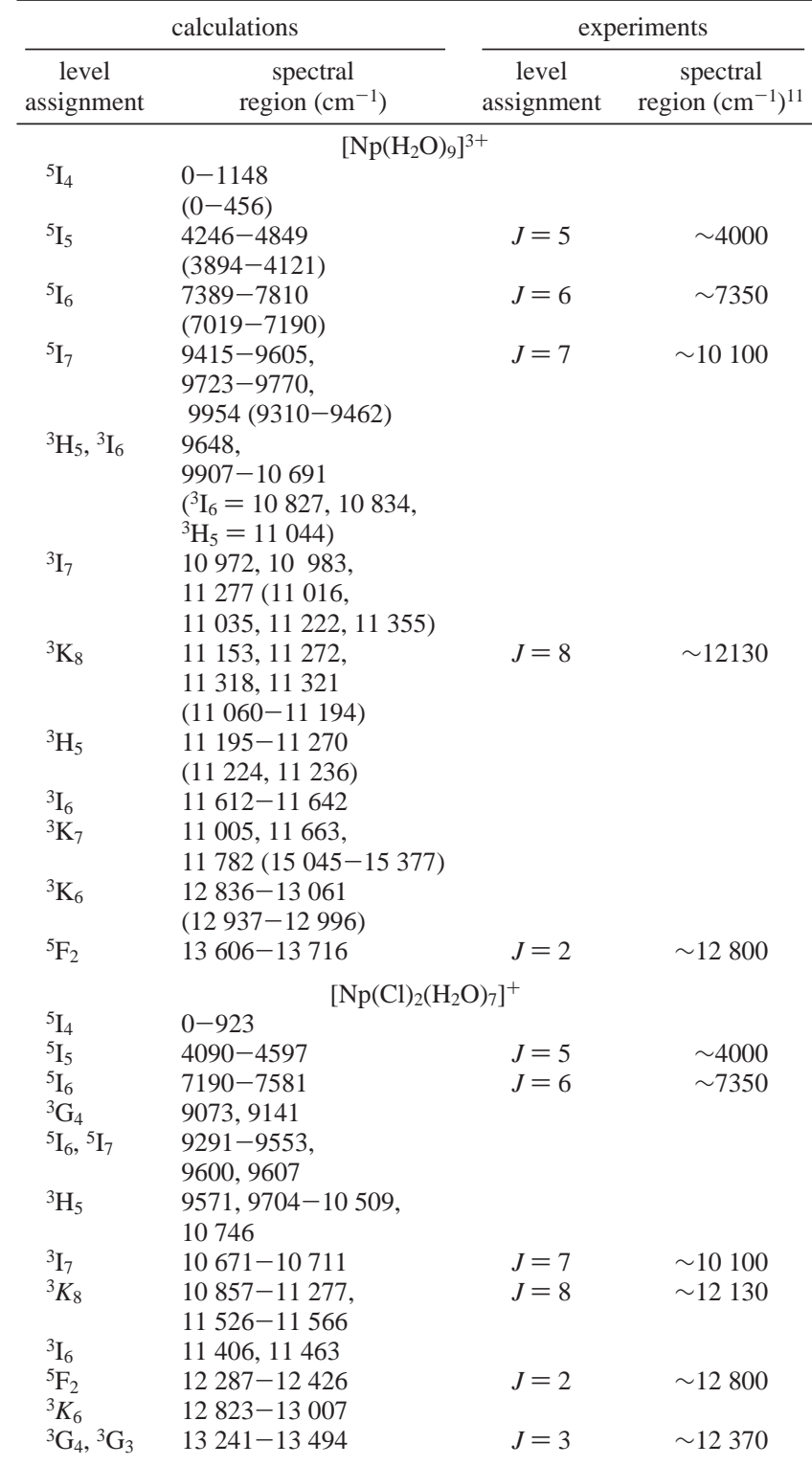

${ }^{a}$ Only data with precise experimental counterparts are listed (see Supporting Information for the whole spectra up to $20000 \mathrm{~cm}^{-1}$ ). Computed values for the free ion $\mathrm{Np}^{3+}$ are indicated in parentheses for comparison with $\left[\mathrm{Np}\left(\mathrm{H}_{2} \mathrm{O}\right)_{9}\right]^{3+}$. For the aquo and chloro complexes, $\mathrm{Np}^{3+}$ in $1.0 \mathrm{M}$ $\mathrm{DClO}_{4}$ at $23{ }^{\circ} \mathrm{C}$, ref 35 .

of one electron with itself. Unfortunately, we must admit that such errors are quite difficult to quantify separately. In particular, the agreement on Pr values is slightly worse than for their actinide counterparts, as suggested by the higher mean square deviation (Table 1). It is however difficult to know where such a discrepancy comes from, especially as a difference on the order of $40 \mathrm{~cm}^{-1}$ is not really meaningful. On the other hand, few experimental references are available in the literature, and they may sometimes be unreliable: uncertainties in $J$ values beyond $10000 \mathrm{~cm}^{-1}$ are clearly admitted, ${ }^{35}$ and experimental coordination numbers are generally unknown. It is worth noting that when more accurate references are available as for $U$ complexes (Table 4), the agreement is much better and continues until $20000 \mathrm{~cm}^{-1}$. Therefore, in Tables 3-6, only clearly described values are
Table 6. Calculated and Experimental Electronic States and Energy Levels of $\left[\mathrm{Pu}\left(\mathrm{H}_{2} \mathrm{O}\right)_{9}\right]^{3+}$ and $\left[\mathrm{Pu}(\mathrm{Cl})_{2}\left(\mathrm{H}_{2} \mathrm{O}\right)_{7}\right]^{+a}$

\begin{tabular}{|c|c|c|c|}
\hline \multicolumn{2}{|r|}{ calculations } & \multicolumn{2}{|c|}{ experiments } \\
\hline $\begin{array}{c}\text { level } \\
\text { assignment }\end{array}$ & $\begin{array}{c}\text { spectral } \\
\text { region }\left(\mathrm{cm}^{-1}\right)\end{array}$ & $\begin{array}{c}\text { level } \\
\text { assignment }\end{array}$ & $\begin{array}{l}\text { spectral } \\
\text { region }\left(\mathrm{cm}^{-1}\right)^{12}\end{array}$ \\
\hline \multicolumn{4}{|c|}{$\left[\mathrm{Pu}\left(\mathrm{H}_{2} \mathrm{O}\right)_{9}\right]^{3+}$} \\
\hline${ }^{4} \mathrm{G}_{5 / 2}$ & $\begin{array}{l}0-333 \\
(0-125)\end{array}$ & & \\
\hline${ }^{6} \mathrm{H}_{7 / 2}$ & $\begin{array}{l}4345-4967 \\
(4116-4307)\end{array}$ & & \\
\hline${ }^{4} \mathrm{G}_{7 / 2}$ & $\begin{array}{l}7186,7211, \\
7334\end{array}$ & & \\
\hline${ }^{6} \mathrm{H}_{9 / 2}$ & $\begin{array}{l}7475-8142 \\
(7329-7497)\end{array}$ & $J=9 / 2$ & $\sim 7000$ \\
\hline${ }^{4} \mathrm{G}_{5 / 2}$ & $\begin{array}{l}8387,8601 \\
(7523-7554)\end{array}$ & & \\
\hline${ }^{4} \mathrm{~K}_{15 / 2}$ & $\begin{array}{l}8813-9510 \\
(9646,9669 \\
9782-10001)\end{array}$ & $J=15 / 2$ & $\sim 11700$ \\
\hline${ }^{4} \mathrm{~K}_{15 / 2}+{ }^{4} \mathrm{I}_{11 / 2}$ & $9617-9741$ & $J=11 / 2$ & 9090 \\
\hline${ }^{6} \mathrm{H}_{11 / 2}$ & 9836-9994 & $J=7 / 2$ & 9900 \\
\hline${ }^{4} \mathbf{I}_{13 / 2}$ & $\begin{array}{l}10060-11096 \\
(9695,9710, \\
10656-10813)\end{array}$ & $J=13 / 2$ & 11070 \\
\hline${ }^{4} \mathrm{G}_{9 / 2}$ & $\begin{array}{l}11137-13183 \\
\left({ }^{4} \mathrm{G}_{5 / 2}=11114-13430\right)\end{array}$ & $\begin{array}{l}J=9 / 2,15 / 2, \\
5 / 2\end{array}$ & $\sim 12500$ \\
\hline \multicolumn{4}{|c|}{$\left[\mathrm{Pu}(\mathrm{Cl})_{2}\left(\mathrm{H}_{2} \mathrm{O}\right)_{7}\right]^{+}$} \\
\hline $\begin{array}{l}{ }^{6} \mathrm{H}_{5 / 2} \\
{ }^{6} \mathrm{H}_{72}\end{array}$ & $0-584$ & & \\
\hline $\begin{array}{l}{ }^{6} \mathrm{H}_{7 / 2} \\
{ }^{4} \mathrm{G}_{7 / 2}\end{array}$ & $\begin{array}{l}3987-6974 \\
6998-7297\end{array}$ & & \\
\hline${ }^{6} \mathrm{H}_{9 / 2}$ & $\begin{array}{l}7412,7665 \\
7709\end{array}$ & $J=9 / 2$ & $\sim 7000$ \\
\hline $\begin{array}{l}{ }^{4} \mathrm{~F}_{5 / 2},{ }^{4} \mathrm{G}_{7 / 2}, \\
{ }^{4} \mathrm{G}_{9 / 2}\end{array}$ & $\begin{array}{l}7556,8190, \\
8335,9012\end{array}$ & & \\
\hline${ }^{6} \mathrm{H}_{11 / 2},{ }^{6} \mathrm{I}_{11 / 2}$ & $9351-9861$ & $J=11 / 2$ & 9090 \\
\hline${ }^{4} \mathrm{~K}_{15 / 2}$ & $9893-10246$ & $J=15 / 2$ & $\sim 11700$ \\
\hline${ }^{6} \mathrm{H}_{11 / 2},{ }^{4} \mathrm{I}_{13 / 2}$ & $10497-10611$ & $J=13 / 2$ & 11070 \\
\hline${ }^{6} \mathrm{G}_{7 / 2}$ & 10728,12824 & $J=7 / 2$ & 9900 \\
\hline${ }^{4} \mathrm{H}_{11 / 2}$ & $10783-11062$ & & \\
\hline${ }^{4} \mathrm{H}_{9 / 2}$ & $13105-13350$ & $\begin{array}{l}J=9 / 2,15 / 2, \\
5 / 2\end{array}$ & $\sim 12500$ \\
\hline
\end{tabular}

${ }^{a}$ Only data with accurate experimental counterparts are listed (see Supporting Information for the whole spectra up to $20000 \mathrm{~cm}^{-1}$ ). Computed values for the free ion $\mathrm{Pu}^{3+}$ are indicated in parentheses for comparison with $\left[\mathrm{Pu}\left(\mathrm{H}_{2} \mathrm{O}\right)_{9}\right]^{3+}$. For the aquo and chloro complexes, $\mathrm{Pu}^{3+}$ in $1.0 \mathrm{M}$ $\mathrm{DClO}_{4}$ at $23{ }^{\circ} \mathrm{C}$, ref 35 .

listed, while the complete spectra are given in Supporting Information.

Despite these differences, the qualitative agreement with experimental values remains very good, in particular the description of covalency effects. The latter are generally quite low for trivalent rare earth systems, in particular for $4 \mathrm{f}$ orbitals that are well-shielded by $5 \mathrm{~s}$ and $5 \mathrm{p}$ levels. Yet, this results in narrow bands that allow the observation of small energetic shifts. The LFDFT programs do not include any information about intensities or bandwidths, but even so, variations between the chloro and aquo complexes are noted. Our computed spectra indeed feature a shift to the red when going from $\left[\mathrm{M}\left(\mathrm{H}_{2} \mathrm{O}\right)_{9}\right]^{3+}$ to $\left[\mathrm{M}(\mathrm{Cl})_{2}\left(\mathrm{H}_{2} \mathrm{O}\right)_{7}\right]^{+}$imputed to a slight increase in covalency within the metal-chloride bond, as quantified in the early 1960 s by Wong et al. ${ }^{36}$ This shift, already observed for $\mathrm{Nd}$ and Am chloro complexes, ${ }^{37,38}$ is

(35) Carnall, W. T.; Wybourne, B. G. J. Chem. Phys. 1964, 40, 3428.

(36) Wong, E. Y.; Stafsudd, O. M.; Johnston, D. R. J. Chem. Phys. 1963, $39,786$.

(37) Choppin, G. R.; Henrie, D. E.; Buijs, K. Inorg. Chem. 1966, 5, 1743 

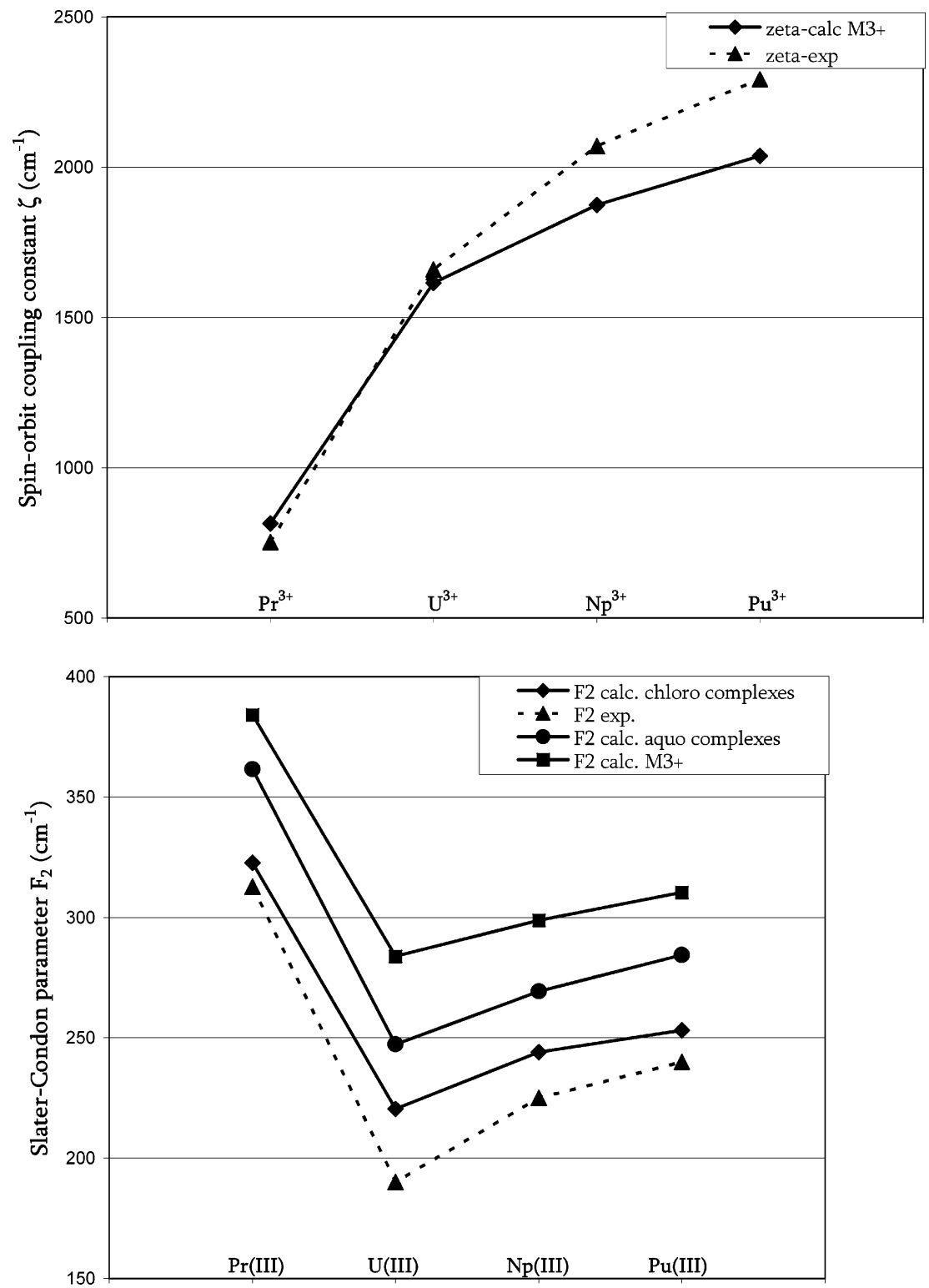

Figure 1. Experimental vs calculated evolutions of the spin-orbit coupling constant $\zeta$ (top) and the Slater-Condon parameter, $F_{2}$ (bottom) in $\mathrm{cm}^{-1}$.

generally referred as the nephelauxetic effect. ${ }^{39}$ As the overlap with the ligand becomes stronger, $f$ orbital expansion increases, and the electrostatic repulsion is thus lowered. The resulting $\mathrm{f}-\mathrm{f}$ transitions generally undergo a reduction in energy, but this trend can be far more complex when strong spin-orbit coupling is involved. Indeed, the comparison of the bare ion spectrum (Tables 3-6) with that of the aquo complex globally shows a displacement to lower energies for $\left[\mathrm{M}\left(\mathrm{H}_{2} \mathrm{O}\right)_{9}\right]^{3+}$, but this effect tends to reverse when moving to heavier elements. Note that such an inversion is also systematically found for the first two or three transitions of each spectrum.

As expected, the nephelauxetic effect remains quite low for praseodymium (Table 3) because of the contraction of its $4 \mathrm{f}$ orbitals. The ${ }^{3} \mathrm{H}_{5}$ and ${ }^{3} \mathrm{~F}_{2}$ bands are reported to be hyper-

(38) Tilson, J. L.; Naleway, C.; Seth, M.; Shepard, R.; Wagner, A. F.; Ermler, W. C. J. Chem. Phys. 2004, 121, 5661.

(39) Choppin, G. R. J. Alloys Compd. 2002, 344, 55. sensitive in the literature, but the absence of experimental data for such states prevents us from any interpretation.

Covalency is logically strengthened in actinide systems because of the expansion of the $5 \mathrm{f}$ orbitals. ${ }^{40,41}$ This has been notably identified in Am(III) complexes of polyaminocarboxylate ligands where larger spectral shifts were recorded than for the lanthanides homologues. ${ }^{42}$ However, the appreciation of the nephelauxetic effect is made difficult because of the higher value of the actinide spin-orbit coupling constant that induces a significant splitting of spectroscopic terms and a strong mixing of $L, S$, and $J$. The spectral analysis is consequently quite complex, especially because experimental assignments are rather uncertain for $\mathrm{Np}$ and $\mathrm{Pu}$. The first part of the U(III) and $\mathrm{Np}$ (III) system spectra nonetheless shows a global red-shift from 100 to 600 $\mathrm{cm}^{-1}$ (374 $\mathrm{cm}^{-1}$ for the ${ }^{4} \mathrm{I}_{9 / 2}$ uranium state or $225 \mathrm{~cm}^{-1}$ for

(40) Krupa, J. C. J. Alloys Compd. 1995, 225, 1.

(41) Liu, G. K. J. Solid State Chem. 2005, 178, 489.

(42) Della Site, A.; Baybarz, R. D. J. Inorg. Nucl. Chem. 1969, 31, 2201. 
the neptunium ${ }^{5} \mathrm{I}_{4}$ counterpart). Such a comparison is almost impossible for $\mathrm{Pu}$ complexes because of the strong mixing between electronic states.

In this regard, more relevant interpretations can be inferred from the calculated parameters sketched in Figure 1 (see precise values in Supporting Information, Table S3). Electrostatic repulsion and spin-orbit (SO) coupling are the two main effects experienced by f electrons; therefore, the SlaterCondon parameter, $F_{2}$, and of the SO constant, $\zeta$, are generally good indicators of the results consistency. The former is strongly dependent on the expansion of the $f$ orbitals, while the latter follows the nucleus charge variations. They should thereby increase along each series, ${ }^{40,41}$ and indeed, their values step up from $\mathrm{U}$ to $\mathrm{Pu}$ as $Z$ becomes higher and $5 \mathrm{f}$ orbitals get more contracted. The spin-orbit coupling constant, $\zeta$, thus goes from $1615 \mathrm{~cm}^{-1}$ for $\mathrm{U}^{3+}$ to 1875 and $2038 \mathrm{~cm}^{-1}$ for $\mathrm{Np}^{3+}$ and $\mathrm{Pu}^{3+}$, respectively. From the lanthanides to the actinides, $F_{2}$ and $\zeta$ behave in an opposite pattern. The lanthanide $\mathrm{f}$ orbitals being more contracted than those of actinides results in a weaker electronic repulsion for An compounds and thus a lower $F_{2}$ value $\left(F_{2}\left(\left[\mathrm{Pr}(\mathrm{H} 2 \mathrm{O})_{9}\right]^{3+}\right)=361.5 \mathrm{~cm}^{-1}\right.$ vs $F_{2}\left(\left[\mathrm{U}(\mathrm{H} 2 \mathrm{O})_{9}\right]^{3+}\right)$ $\left.=247.3 \mathrm{~cm}^{-1}\right)$. At the same time, $\zeta$ is logically calculated to be twice as large for $\mathrm{U}^{3+}\left(1615 \mathrm{~cm}^{-1}\right)$ than it is for $\operatorname{Pr}^{3+}$ $\left(814 \mathrm{~cm}^{-1}\right)$ because of the increasing charge of the nucleus.

From a more quantitative point of view, the calculated parameters are close to the experimental ones. $\zeta$ is on average $\left(140 \mathrm{~cm}^{-1}\right)$ too high with discrepancies going from $2.7\left(\mathrm{U}^{3+}\right)$ to $11.1 \%\left(\mathrm{Pu}^{3+}\right)$. This time, experimental data cannot be blamed because, with the same reference, the value for $\mathrm{U}^{3+}$ matches well $\left(1666 \mathrm{~cm}^{-1}\right) . F_{2}$ is always found to be higher than its experimental counterpart $\left(|\Delta E| \approx 30 \mathrm{~cm}^{-1}\right)$, which confirms that the underestimation of the $\mathrm{M}-\mathrm{Cl}$ distances has a low impact on the LFDFT results. As already discussed, this discrepancy may be linked to the self-interaction error. Other Slater integrals $\left(F_{4}\right.$ and $\left.F_{6}\right)$ appear almost unaffected with discrepancies smaller than few per centimeter (see Table S3). Ferguson and Wood $^{43}$ have discussed these variations

(43) Ferguson, J.; Wood, D. L. Aust. J. Chem. 1970, 23, 861.

(44) David, F.; Fourest, B.; Hubert, S.; Le Du, J.-F.; Revel, R.; Den Auwer, C.; Madic, C.; Morss, L. R.; Ionova, G.; Mikhalko, V.; Vokhmin, V.; Nikonov, M.; Berthet, J. C.; Ephritikhine, M. In Speciation, Techniques and Facilities for Radioactive Materials at Synchrotron Light Sources; Nuclear Energy Agency, Organisation for Economic Co-Operation and Development: Grenoble, France 1998; pp 95-100.

(45) Carnall, W. T.; Fields, P. R.; Rajnak, K. J. Chem. Phys. 1968, 49 , 4412.

(46) Carnall, W. T.; Fields, P. R.; Rajnak, K. J. Chem. Phys. 1968, 49, 4424.

(47) Keller, B.; Legendziewicz, J.; Glinski, J.; Samela, S. J. Alloys Compd. 2000, 300-301, 334.

(48) Keller, B.; Legendziewicz, J.; Glinski, J. Spectrosc. Chim. Acta A 1998, $54,2207$.

(49) Katzin, L. I.; Barnett, M. L. J. Phys. Chem. 1964, 68, 3779. in terms of spatial electronic areas. $F_{2}$ reflects outer properties, and its reduction therefore provides a measure of covalency, as is clearly shown when comparing $F_{2}$ values for the bare ion with that for coordinated species. $F_{4}$ and $F_{6}$ deal with the inner regions. The $F_{4} / F_{2}$ ratio is hence commonly used to assess the nephelauxetic effect. One can indeed note in Table S3 that corresponding values are, with the exception of U systems, systematically lower for chloro complexes $(\sim 0.12)$ than for the aquo ones $(\sim 0.10)$ and that they are similar from one metal to another. This variation is logically caused by outer effects since $F_{4}$ is almost independent of the surrounding ligands. In the case of U(III), the slight decrease of the $F_{4}$ value explains that $F_{4} / F_{2}$ does not change, while the drop of $F_{2}$ clearly indicates the increase in covalency.

\section{Conclusion}

The LFDFT method has been successfully applied to the calculation of the $\mathrm{f}-\mathrm{f}$ transitions for aquo and chloro complexes of trivalent $\mathrm{Pr}, \mathrm{U}, \mathrm{Np}$, and $\mathrm{Pu}$. The results are found to be in good agreement with experimental data, at least for the lowest bands. Characteristic assignments are always identified, but inaccuracies in experimental assignments, combined with differences between theoretical and experimental conditions, may play a significant role in the reported discrepancies. Despite this, the nephelauxetic effect has been clearly shown: when moving from aquo to chloro complexes, spectral bands are displaced to higher wavelengths by several hundred per centimeter, while the experimental counterparts are somewhat lower. These covalency effects are confirmed by the decrease in the $F_{2}$ SlaterCondon parameter. Our ultimate purpose being to get information on the chloride coordination number and to unravel the experimental contradiction between spectral and EXAFS data, we plan to calculate the environment-sensitive $\mathrm{f}-\mathrm{d}$ transitions.

Acknowledgment. This study was supported by the COST Short Term Scientific Mission program (action D26) and the Swiss National Science Foundation. L.P would like to thank all the chemistry department staff of the University of Fribourg for their kind hospitality.

Supporting Information Available: Complete calculated $\mathrm{f}-\mathrm{f}$ transitions for the $\mathrm{Np}(\mathrm{III})$ and $\mathrm{Pu}(\mathrm{III})$ aquo and chloro complexes (Tables S1 and S2), as well as the values for the fitted parameters (Table S3). This material is available free of charge via the Internet at http://pubs.acs.org.

IC060149X

(50) Drozdzynski, J. J. Inorg. Nucl. Chem. 1978, 40, 319.

(51) Caspers, H. H.; Buchanan, R. A. J. Chem Phys. 1965, 43, 2124

(52) (a) Sugar, J. Phys. Rev. 1965, 14, 731. (b) Crosswhite, H. M.; Diecke, G. H.; Carter, W. J. J. Chem. Phys. 1965, 43, 2047. 